\title{
Fully differential vector-boson fusion Higgs pair production at next-to-next-to-leading order
}

\author{
Frédéric A. Dreyer ${ }^{1}$ and Alexander Karlberg ${ }^{2}$ \\ ${ }^{1}$ Rudolf Peierls Centre for Theoretical Physics, University of Oxford, Clarendon Laboratory, \\ Parks Road, Oxford OX1 3PU, United Kingdom \\ ${ }^{2}$ Department of Physics, University of Zürich, CH-8057 Zürich, Switzerland
}

(Received 23 November 2018; published 29 April 2019)

\begin{abstract}
We calculate the fully differential next-to-next-to-leading order (NNLO) QCD corrections to vectorboson fusion (VBF) Higgs pair production. This calculation is achieved in the limit in which there is no colored cross-talk between the colliding protons, using the projection-to-Born method. We present differential cross sections of key observables, showing corrections of up to $3 \%-4 \%$ at this order after typical VBF cuts, with the total cross section receiving contributions of about $2 \%$. In contrast to single Higgs VBF production, we find that the NNLO corrections are for the most part within the next-to-leading order scale uncertainty bands.
\end{abstract}

DOI: $10.1103 /$ PhysRevD.99.074028

\section{INTRODUCTION}

Since the 2012 discovery of the Higgs boson [1,2], one of the focuses of the experimental program of the Large Hadron Collider (LHC) has been the measurement of its couplings to fermions, to other bosons, and to itself. The self-coupling of the Higgs boson is of particular interest to understand the electroweak symmetry breaking mechanism, and to constrain new physics beyond the standard model (SM). In that context, Higgs pair production plays a key role at the LHC [3-15], its high luminosity upgrade (HL-LHC), and at future hadron colliders in improving our understanding of the Higgs sector.

In this article, we focus on double Higgs production via VBF, shown in Fig. 1, which is the second largest channel at the LHC after gluon-gluon fusion [16]. The VBF production mode is of particular interest in di-Higgs production: due to the presence of two tagging jets, one can significantly reduce the large backgrounds through an appropriate choice of cuts; it also provides a unique sensitivity to deviations from the SM in the trilinear Higgs coupling [17], and is the most promising channel for measurements of the $h h V V$ quartic coupling at the LHC [18].

Because of the important role that double Higgs production via VBF plays at the LHC and beyond, it is crucial that precise predictions for its production rate can be

Published by the American Physical Society under the terms of the Creative Commons Attribution 4.0 International license. Further distribution of this work must maintain attribution to the author(s) and the published article's title, journal citation, and DOI. Funded by SCOAP ${ }^{3}$. achieved. So far the differential cross section has been calculated up to next-to-leading order (NLO) $[17,19,20]$, and up to next-to-next-to-next-to-leading order $\left(\mathrm{N}^{3} \mathrm{LO}\right)$ in the structure function approach [21] where all hadronic radiation is integrated over $[22,23]$.

Since cuts on the tagging jets are critical in reducing the large experimental backgrounds, having access to the jet kinematics is necessary to produce realistic predictions. In particular, higher order calculations of single-Higgs VBF production found that while corrections to the inclusive cross section were small $[24,25]$, VBF cuts can substantially impact the structure of these contributions [26,27].

We present for the first time the calculation of di-Higgs production to next-to-next-to-leading order (NNLO) in perturbative QCD for differential cross sections, which is also the first of this type for a $2 \rightarrow 4$ process. This brings the VBF Higgs pair production channel to the same accuracy as double Higgs gluon-gluon fusion production $[28,29]$.

These results are achieved using the deep inelastic scattering (DIS) approximation, which corresponds to the limit in which there is no colored cross-talk between the two colliding protons. This approximation is exact at NLO, where the gluon exchange between the protons is null for color reasons, and has been shown to be accurate to more than $1 \%$ at NNLO [30-32] for single Higgs VBF production. Because the presence of an additional Higgs boson does not impact the color flow between the hadrons, this limit is expected to be just as accurate for Higgs pair production. The approximation also corresponds to the exact calculation for two sectors with different copies of QCD which interact purely through the weak force, shown in different colors in Fig. 1. 


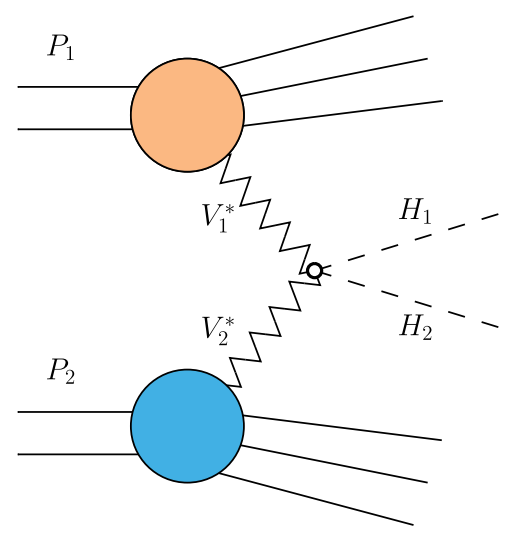

FIG. 1. Diagram of vector-boson fusion (VBF) Higgs pair production, with the colored objects representing the incoming protons.

\section{CALCULATION SETUP}

The VBF Higgs pair production cross section is calculated as a double DIS process, and can be expressed as [21]

$$
\begin{aligned}
d \sigma= & \sum_{V} \frac{2 G_{F}^{4} m_{V}^{8}}{s} \Delta_{V}^{2}\left(Q_{1}^{2}\right) \Delta_{V}^{2}\left(Q_{2}^{2}\right) d \Omega_{\mathrm{VBF}} \\
& \times \mathcal{W}_{\mu \nu}^{V}\left(x_{1}, Q_{1}^{2}\right) \mathcal{M}^{V, \mu \rho} \mathcal{M}^{V *, \nu \sigma} \mathcal{W}_{\rho \sigma}^{V}\left(x_{2}, Q_{2}^{2}\right) .
\end{aligned}
$$

Here $V=W^{ \pm}, Z$ is the mediating boson, $G_{F}$ is Fermi's constant, $m_{V}$ is the mass of the vector boson, $\sqrt{s}$ is the collider center-of-mass energy, $\Delta_{V}^{2}$ is the squared boson propagator, $Q_{i}^{2}=-q_{i}^{2}$ and $x_{i}=Q_{i}^{2} /\left(2 P_{i} \cdot q_{i}\right)$ are the usual DIS variables, $\mathcal{W}_{\mu \nu}^{V}$ is the hadronic tensor and $d \Omega_{\mathrm{VBF}}$ is the four particle VBF phase space. The matrix element of the vector-boson fusion subprocess is given by [33]

$$
\begin{aligned}
\mathcal{M}^{V, \mu \nu}= & {\left[\left(1+\frac{4 m_{V}^{2}}{\left(q_{1}+k_{1}\right)^{2}-m_{V}^{2}+i \Gamma_{V} m_{V}}\right.\right.} \\
& \left.+\frac{6 \nu \lambda}{\left(k_{1}+k_{2}\right)^{2}-m_{H}^{2}+i \Gamma_{H} m_{H}}\right) g^{\mu \nu} \\
& +\frac{m_{V}^{2}}{\left(q_{1}+k_{1}\right)^{2}-m_{V}^{2}+i \Gamma_{V} m_{V}} \\
& \left.\times \frac{\left(2 k_{1}^{\mu}+q_{1}^{\mu}\right)\left(k_{2}^{\nu}-k_{1}^{\nu}-q_{1}^{\nu}\right)}{m_{V}^{2}-i \Gamma_{V} m_{V}}\right]+\left(k_{1} \leftrightarrow k_{2}\right),
\end{aligned}
$$

where $k_{1}$ and $k_{2}$ are the Higgs momenta such that $q_{1}+q_{2}=k_{1}+k_{2}, \lambda$ is the $\mathrm{SM}$ trilinear Higgs selfcoupling, $\nu$ is the vacuum expectation value of the Higgs field, and we have absorbed a factor $\sqrt{2} G_{F} m_{V}^{2}$ from $\mathcal{M}^{V}$ into the overall normalization of the cross section.

To obtain differential results, we use the projection-toBorn method [26], combining an inclusive NNLO calculation with a calculation at NLO of two Higgs bosons in association with three jets. The electroweak di-Higgs three jet process was obtained by modifying the matrix elements in POWHEG's corresponding single-Higgs process $[34,35]$. We cross-checked this implementation by verifying that the kinematical properties of the third and fourth jets agreed within statistical uncertainties with predictions obtained through the MadGraph5_aMC@NLO [36] framework. We also compared our results at NLO with VBFNLO [37], finding agreement at the few permille level.

For our predictions, we consider $14 \mathrm{TeV}$ proton-proton collisions, with the PDF4LHC15_nnlo_mc [38] set as implemented in LHAPDF version 6.1.6 [39], a Higgs mass $m_{H}=125 \mathrm{GeV}$, and electroweak parameters fixed by $m_{W}=80.379 \mathrm{GeV}, m_{Z}=91.1876 \mathrm{GeV}$, and $G_{F}=$ $1.16637 \times 10^{-5} \mathrm{GeV}^{-2}$ consistent with the most recent review of particle physics [40]. For internal propagators we use $\Gamma_{Z}=2.4952 \mathrm{GeV}, \Gamma_{W}=2.141 \mathrm{GeV}$, and $\Gamma_{H}=$ $4.030 \times 10^{-3} \mathrm{GeV}$, while the final state Higgs bosons are considered in the narrow-width approximation. The central scale is chosen analogously to the one considered in the single-Higgs NNLO calculation [26]

$$
\mu_{0}^{2}\left(p_{t, H H}\right)=\frac{m_{H}}{2} \sqrt{\left(\frac{m_{H}}{2}\right)^{2}+p_{t, H H}^{2}},
$$

motivated by the fact that it approximates $\sqrt{Q_{1}^{2} Q_{2}^{2}}$ relatively well. The uncertainties from missing higher orders are estimated by simultaneously varying the renormalization and factorization scale up and down by a factor of 2 .

The events have to pass a set of VBF selection cuts. We require at least two hard jets with

$$
p_{t, j}>25 \mathrm{GeV}, \quad\left|y_{j}\right|<4.5 \text {. }
$$

The two hardest jets additionally have to satisfy

$$
m_{j_{1}, j_{2}}>600 \mathrm{GeV}, \quad\left|y_{j_{1}}-y_{j_{2}}\right|>4.5, \quad y_{j_{1}} y_{j 2}<0 .
$$

We define our jets using the anti- $k_{t}$ algorithm [41] as implemented in FastJet version 3.3.0 [42]. The jet radius is set to $R=0.4$.

\section{RESULTS}

The total cross sections before and after VBF cuts are given in Table I. The errors given correspond to the envelope of the three-point scale variation, while the statistical uncertainties are negligible. We can observe here that the convergence of the inclusive cross section is better than that of the fiducial cross section, with a second order correction of only $2 \%$, while it is of about $1.7 \%$ after cuts. At NNLO, the scale variation uncertainty on the fiducial cross section is reduced by a factor of 4 , to less than $1 \%$. This stands in contrast to the single-Higgs process, where there is almost no reduction in scale uncertainties at NNLO, and where the NLO variation bands do not encompass the 
TABLE I. Cross sections at LO, NLO and NNLO for VBF Higgs production, fully inclusively and with VBF cuts. The uncertainties are obtained from a three-point scale variation. The statistical uncertainty on the inclusive and fiducial cross sections are below one permille.

\begin{tabular}{lcc}
\hline \hline & $\sigma^{\text {(no cuts) }}[\mathrm{fb}]$ & $\sigma^{\text {(VBF cuts) }}[\mathrm{fb}]$ \\
\hline LO & $2.016_{-0.142}^{+0.164}$ & $0.799_{-0.069}^{+0.082}$ \\
NLO & $2.049_{-0.021}^{+0.007}$ & $0.726_{-0.020}^{+0.005}$ \\
NNLO & $2.053_{-0.003}^{+0.000}$ & $0.713_{-0.001}^{+0.004}$ \\
\hline \hline
\end{tabular}

central value of the NNLO prediction. One reason for this can likely be found in the different kinematics probed in di-Higgs VBF production. At leading order the two tagging jets take the full recoil of the two fusing vector bosons. Since twice as much energy is required to produce two Higgs bosons compared to one, we expect the jets in diHiggs VBF production to be harder than the jets in single Higgs VBF production. For that reason more events pass the VBF cuts even when they radiate outside of the jet cone.

In Fig. 2, we present the differential distributions after VBF cuts of the Higgs bosons. The Higgs bosons are ordered in transverse momentum, with the harder one labeled $H_{1}$, and the softer boson $H_{2}$. We show the transverse momentum of both Higgs bosons, $p_{t, H_{1}}$ and $p_{t, H_{2}}$, as well as the rapidity and invariant mass of the Higgs pair, $y_{H H}$ and $m_{H H}$. We see that the NNLO corrections can be sizeable, at about $4 \%$, in the region where both Higgs bosons are soft, while for Higgs bosons with large transverse momentum the corrections become quite small, in the $1 \%-2 \%$ range. The invariant mass of the Higgs pair is an observable of particular interest, as its distribution can be very sensitive to deviations from the SM in certain models $[18,43,44]$. Here we can observe moderate NNLO corrections, up to about $2 \%$, albeit with some kinematical dependence.

Differential cross sections after VBF cuts for the tagging jets are shown in Fig. 3. Here it is interesting to see that the structure of the NNLO corrections is quite different than in the single-Higgs process. In particular, they are as for the fiducial cross section smaller and mostly contained within the scale variation bands of the NLO predictions. The transverse momentum of the two tagging jets receives corrections of $2 \%-4 \%$, with the corrections being more pronounced at moderate $p_{t}$ values. For the rapidity separation between the jets and their invariant mass, the corrections are of about $2 \%$, with little dependence on the observable, except at small values of the rapidity separation, where the corrections become negligible.

As we pointed out earlier, the tagging jets are expected to be harder than in single-Higgs production, a fact which is confirmed by comparing the slopes of the two leading jets
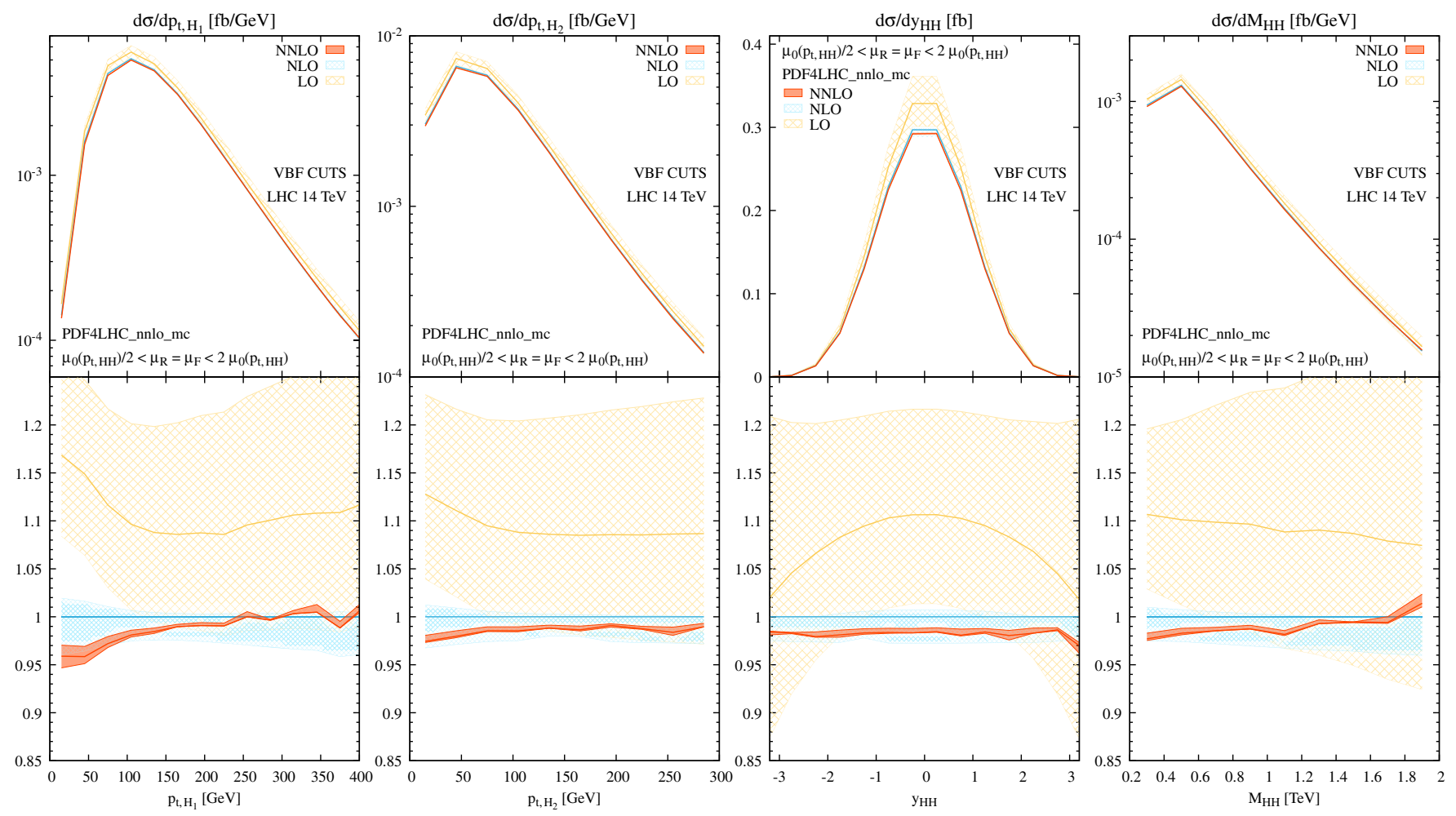

FIG. 2. From left to right, differential cross sections for the transverse momentum distributions for the two Higgs bosons, $p_{t, H_{1}}$ and $p_{t, H_{2}}$, and the distribution of the rapidity and invariant mass of the Higgs pair, $y_{H H}$ and $m_{H H}$. The lower panel shows the ratio to the NLO prediction. 

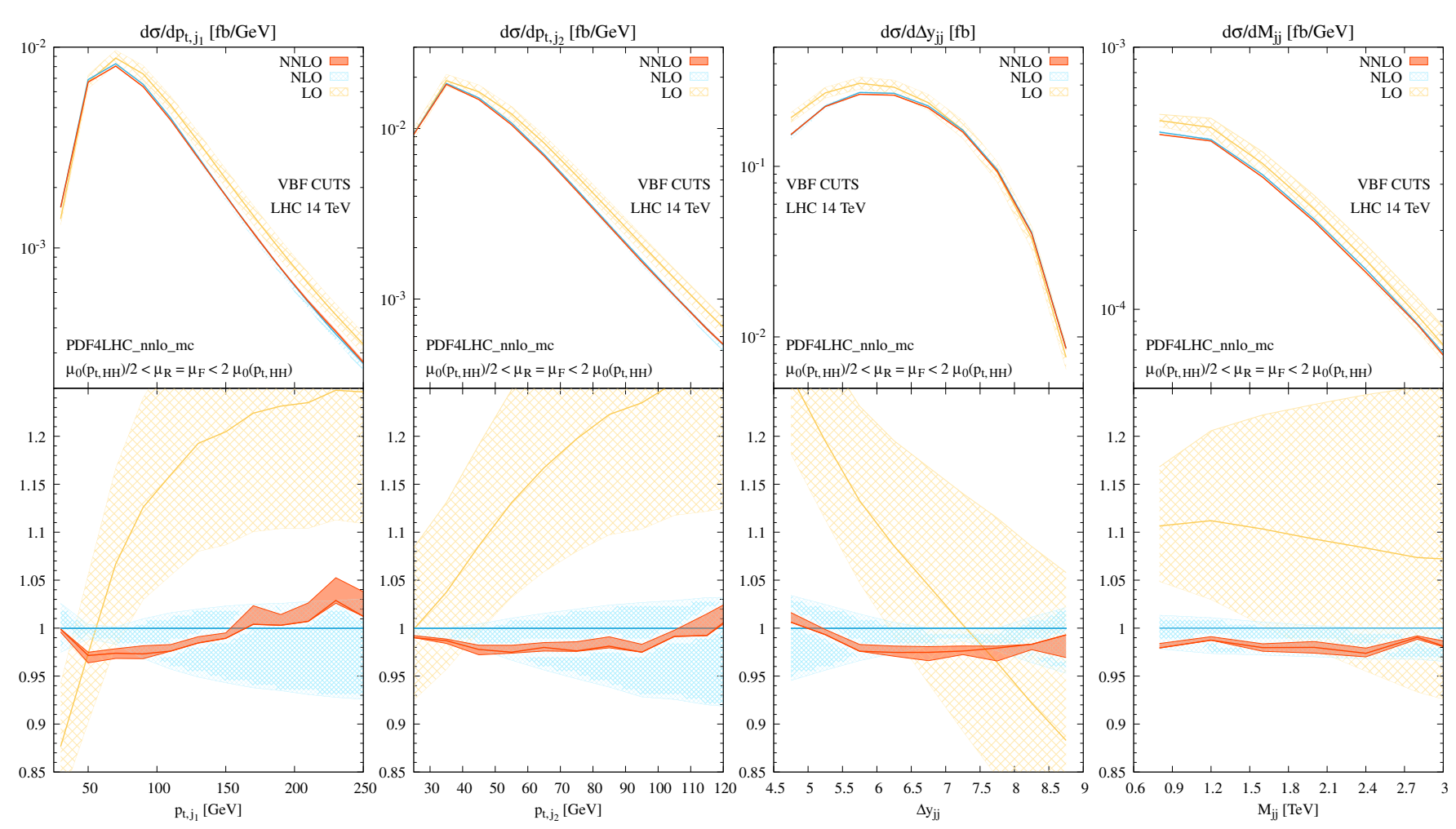

FIG. 3. From left to right, differential cross sections for the transverse momentum distributions for the two leading jets, $p_{t, j_{1}}$ and $p_{t, j_{2}}$, and the distribution for the rapidity and azimuth separation between the two leading jets, $\Delta y_{j_{1}, j_{2}}$ and $m_{j_{1}, j_{2}}$. The lower panel shows the ratio to the NLO prediction.

for both processes. We find that in single-Higgs production the slope of the transverse momentum of the two hardest jets falls more steeply. This partly explains the difference in the structure of the higher order QCD corrections between single- and di-Higgs production.

\section{OTHER CONTRIBUTIONS}

We note that there are theoretical effects beyond the perturbative QCD corrections considered in this article. We have estimated the contribution of the $s$-channel, which is not included in the DIS approximation using MadGraph5_aMC@NLO version 2.6.2. While it has a substantial effect on the inclusive cross section, of about $27 \%$, it is reduced to one permille after our VBF cuts are applied and can thus be neglected. There are also nonfactorizable contributions to VBF which first appear at NNLO and are neglected in the DIS approximation. These should, as in the case of single-Higgs production, contribute to less than one percent of the cross section [30-32].

There are also corrections due to higher order electroweak effects. They are currently unknown, but can be estimated from dominant light quark induced channels using Recola (Collier)+MoCaNLO [45-48] for the di-Higgs and single-Higgs VBF process, comparing the latter to HAWK [49]. For di-Higgs VBF production the electroweak (EW) corrections lie between $-5 \%$ and $-7 \%$ for both the inclusive and fiducial cross sections. Compared to the fiducial singleHiggs VBF prediction of roughly $-8.5 \%$ (using the same setup, i.e., excluding photonic and b-quark channels) the diHiggs VBF process thus does not seem to receive large VBS-like corrections [50,51]. The full electroweak corrections should therefore contribute at about the same level as the NNLO QCD corrections reported here.

Cross sections after cuts are also affected by nonperturbative effects. Using Pythia version 8.219 [52] with the default $4 \mathrm{C}$ tune, we evaluate the correction to the fiducial cross section due to hadronization to about $-1 \%$, while the underlying event leads to a change of $+4 \%$ to the cross section after cuts.

Finally, there are uncertainties associated with the determination of parton distribution functions and the strong coupling constant. Using the PDF4LHC_nnlo_ mc_pdfas set, we find these to be of $2.1 \%$ on the inclusive cross section.

\section{CONCLUSIONS}

In this paper we presented the first differential calculation of NNLO QCD corrections to VBF Higgs pair production. The calculation is fully differential in the kinematics of the tagging jets, allowing for precise predictions of fiducial cross sections. This was achieved by using the projection-to-Born method, combining an inclusive NNLO calculation with a 
differential NLO calculation with an additional final state jet. We showed that after typical VBF cuts, the NNLO corrections are moderate, with corrections of $1.7 \%$ to the total cross section and up to $4 \%$ in some differential observables. The corrections can have a nontrivial kinematic dependence, and are notably different from the single-Higgs production mode.

The full code used for this calculation is available as part of proVBFHH v1.0.0 [53], along with a lightweight program, proVBFH-incl v2.0.0, which allows for the calculation of inclusive cross sections up to $\mathrm{N}^{3} \mathrm{LO}$ for both single and double Higgs production.

\section{ACKNOWLEDGMENTS}

We thank Michael Rauch for collaboration in the early stages of this project and helpful feedback on VBFNLO, and
Marc Zaro for discussions about MadGraph5_aMC@NLO. We are grateful to Jean-Nicolas Lang and Mathieu Pellen for providing us with an estimate of the electroweak corrections. We also wish to thank Gavin Salam, Giulia Zanderighi, and Markus Ebert for valuable comments on the manuscript. F. D. thanks the University of Zurich and the Pauli Center for Theoretical Studies, and A. K. thanks the University of Oxford and the Rudolf Peierls Centre for Theoretical Physics for hospitality while this work was being completed. F. D. is supported by the Science and Technology Facilities Council (STFC) under Grant No. ST/P000770/1. A. K. is supported by the Swiss National Science Foundation (SNF) under Grant No. 200020-175595.
[1] G. Aad et al. (ATLAS Collaboration), Phys. Lett. B 716, 1 (2012).

[2] S. Chatrchyan et al. (CMS Collaboration), Phys. Lett. B 716, 30 (2012).

[3] M. Aaboud et al. (ATLAS Collaboration), Phys. Rev. Lett. 121, 191801 (2018).

[4] M. Aaboud et al. (ATLAS Collaboration), Eur. Phys. J. C 78, 1007 (2018).

[5] M. Aaboud et al. (ATLAS Collaboration) J. High Energy Phys. 11 (2018) 040.

[6] M. Aaboud et al. (ATLAS Collaboration) J. High Energy Phys. 01 (2019) 030.

[7] M. Aaboud et al. (ATLAS Collaboration), Phys. Rev. D 94, 052002 (2016).

[8] G. Aad et al. (ATLAS Collaboration), Phys. Rev. D 92, 092004 (2015).

[9] G. Aad et al. (ATLAS Collaboration), Eur. Phys. J. C 75, 412 (2015).

[10] G. Aad et al. (ATLAS Collaboration), Phys. Rev. Lett. 114, 081802 (2015).

[11] A. M. Sirunyan et al. (CMS Collaboration), arXiv:1810 .11854 .

[12] A. M. Sirunyan et al. (CMS Collaboration) Phys. Lett. B 788, 7 (2019).

[13] A. M. Sirunyan et al. (CMS Collaboration), J. High Energy Phys. 01 (2018) 054.

[14] A. M. Sirunyan et al. (CMS Collaboration), Phys. Lett. B 778, 101 (2018).

[15] A. M. Sirunyan et al. (CMS Collaboration), Phys. Rev. D 96, 072004 (2017).

[16] D. de Florian et al. (LHC Higgs Cross Section Working Group), arXiv: 1610.07922 .

[17] J. Baglio, A. Djouadi, R. Gröber, M. M. Mühlleitner, J. Quevillon, and M. Spira, J. High Energy Phys. 04 (2013) 151.

[18] F. Bishara, R. Contino, and J. Rojo, Eur. Phys. J. C 77, 481 (2017).
[19] T. Figy, Mod. Phys. Lett. A 23, 1961 (2008).

[20] R. Frederix, S. Frixione, V. Hirschi, F. Maltoni, O. Mattelaer, P. Torrielli, E. Vryonidou, and M. Zaro, Phys. Lett. B 732, 142 (2014).

[21] T. Han, G. Valencia, and S. Willenbrock, Phys. Rev. Lett. 69, 3274 (1992).

[22] L.-S. Ling, R.-Y. Zhang, W.-G. Ma, L. Guo, W.-H. Li, and X.-Z. Li, Phys. Rev. D 89, 073001 (2014).

[23] F. A. Dreyer and A. Karlberg, Phys. Rev. D 98, 114016 (2018).

[24] P. Bolzoni, F. Maltoni, S.-O. Moch, and M. Zaro, Phys. Rev. Lett. 105, 011801 (2010).

[25] F. A. Dreyer and A. Karlberg, Phys. Rev. Lett. 117, 072001 (2016).

[26] M. Cacciari, F. A. Dreyer, A. Karlberg, G. P. Salam, and G. Zanderighi, Phys. Rev. Lett. 115, 082002 (2015); 120, 139901(E) (2018).

[27] J. Cruz-Martinez, T. Gehrmann, E. W. N. Glover, and A. Huss, Phys. Lett. B 781, 672 (2018).

[28] D. de Florian and J. Mazzitelli, Phys. Rev. Lett. 111, 201801 (2013).

[29] D. de Florian, M. Grazzini, C. Hanga, S. Kallweit, J. M. Lindert, P. Maierhöfer, J. Mazzitelli, and D. Rathlev, J. High Energy Phys. 09 (2016) 151.

[30] M. Ciccolini, A. Denner, and S. Dittmaier, Phys. Rev. D 77, 013002 (2008).

[31] R. V. Harlander, J. Vollinga, and M. M. Weber, Phys. Rev. D 77, 053010 (2008).

[32] P. Bolzoni, F. Maltoni, S.-O. Moch, and M. Zaro, Phys. Rev. D 85, 035002 (2012).

[33] A. Dobrovolskaya and V. Novikov, Z. Phys. C 52, 427 (1991).

[34] S. Alioli, P. Nason, C. Oleari, and E. Re, J. High Energy Phys. 06 (2010) 043.

[35] B. Jäger, F. Schissler, and D. Zeppenfeld, J. High Energy Phys. 07 (2014) 125. 
[36] J. Alwall, R. Frederix, S. Frixione, V. Hirschi, F. Maltoni, O. Mattelaer, H. S. Shao, T. Stelzer, P. Torrielli, and M. Zaro, J. High Energy Phys. 07 (2014) 079.

[37] J. Baglio et al., arXiv:1107.4038.

[38] J. Butterworth et al., J. Phys. G 43, 023001 (2016).

[39] A. Buckley, J. Ferrando, S. Lloyd, K. Nordström, B. Page, M. Rüfenacht, M. Schönherr, and G. Watt, Eur. Phys. J. C 75, 132 (2015).

[40] M. Tanabashi et al. (Particle Data Group), Phys. Rev. D 98, 030001 (2018).

[41] M. Cacciari, G. P. Salam, and G. Soyez, J. High Energy Phys. 04 (2008) 063.

[42] M. Cacciari, G. P. Salam, and G. Soyez, Eur. Phys. J. C 72, 1896 (2012).

[43] D. B. Kaplan and H. Georgi, Phys. Lett. 136B, 183 (1984).

[44] R. Contino, C. Grojean, M. Moretti, F. Piccinini, and R. Rattazzi, J. High Energy Phys. 05 (2010) 089.

[45] S. Actis, A. Denner, L. Hofer, J.-N. Lang, A. Scharf, and S. Uccirati, Comput. Phys. Commun. 214, 140 (2017).
[46] A. Denner, S. Dittmaier, and L. Hofer, Comput. Phys. Commun. 212, 220 (2017).

[47] R. Feger and M. Pellen (private communication).

[48] J. R. Andersen et al., in 10th Les Houches Workshop on Physics at TeV Colliders (PhysTeV 2017) Les Houches, France, 2017 (2018), http://lss.fnal.gov/archive/2018/conf/ fermilab-conf-18-122-cd-t.pdf.

[49] A. Denner, S. Dittmaier, S. Kallweit, and A. Mück, Comput. Phys. Commun. 195, 161 (2015).

[50] B. Biedermann, A. Denner, and M. Pellen, Phys. Rev. Lett. 118, 261801 (2017).

[51] B. Biedermann, A. Denner, and M. Pellen, J. High Energy Phys. 10 (2017) 124.

[52] T. Sjöstrand, S. Ask, J. R. Christiansen, R. Corke, N. Desai, P. Ilten, S. Mrenna, S. Prestel, C. O. Rasmussen, and P.Z. Skands, Comput. Phys. Commun. 191, 159 (2015).

[53] proVBFHH v1.0.0, http://provbfh.hepforge.org/. 